\title{
Index Volume 2
}

(Numbers followed by asterisk refer to Society Abstracts)

Abelson, H. 271

ABILDGaARD, G. F. 311*

Abortion 291*

Abrams, C. A. L. 43

Absorption, intestinal 301*, 312*

$\mathrm{N}$-Acetylneuraminic acid 125

Achondroplasia 295*

Acid-Base metabolism 416*, 421*, $430^{*}, 431^{*}$

Acidosis 193, 395*, 399*, 408*, 519

- metabolic 295*,303*

Ackerman, G. L. $321 *$

Acrodermatitis enteropathica $301^{*}$

ACTH 501

- effects $306^{*}$

Adam, P. A. J. 399*, 422*

Adamsons, K. 399*, 431*

Addison's disease 501

Adenovirus 288*

Adhikarx, P. K. 288*

Adrenal cortex, hyperplasia $306 *$

Adrenal gland $306^{*}, 456,501$

Adrenergic blockade $300 *$

Aeberhardt, E. 305*

Agammaglobulinemia 72, 209, 300*

Albreaht, I. 320*

Albumin 318*

Aldosterone 22, 456, 501

Alexander, H.E. 410*

Alkalosis, respiratory $303 *$

AlLEN, T. 317*

Allograft rejection 7

Alpert, J. 392*

Altemeier, W. A., III 298*

Al-Ubaidi, Y.Y. $320^{*}$

Amino acids $131,187,493$

Aminoaciduria $302 *, 320 *, 403 *$, 408*, 525

Aminogram 187, 302*

Aminonucleoside $320^{*}$

Aminopterin 306*

Amniotic fluid $318^{*}$

Amos, D. B. 299*

Amphotericin B 299*

Anaphylaxis $300 *$

ANDO, T. 254

Anemia 179, 417*

- hemolytic $302 *, 311 *, 313 *$

- megaloblastic 312*

- nutritional $311 *$

- sickle cell 296*

Angiocardiography 287*

Angiotensin 22

ANGLE, G. R. 421 *

Anoxia 125

Anthony, B.F. 409*

Antibody 298*

- hemagglutinating 38

- secretory $407^{*}$
Anticoagulant $311^{*}$

Anticoagulation therapy $319 *$

Antihemophiliac globulin $313 *$

Anti-Streptolysin-O 409*

Antitrypsin 298*

Anus, imperforate 297*

Apgar score 243

Arachidonic acid 301*

Arginine 94, 525

Artery

- pulmonary 288*

- umbilical $317 *$

ArthuR, J.H. 314*

Ascaris suum 296*

Aspartate carbamyl transferase 307*

Asphyxia 395*, 431*

Atherosclerosis 292*

Auerbach, V.H. 295*

Autoimmunity 7

Avery, M.E. 317*

Avruskin, T. W. $310^{*}$

Ayour, E. M. 409*

Azathioprine 321*

Azotemia 319*

BAGHOFEN, H. 119

Bacteriolysis 315*

BAKER, L. 300*

BARCAI, A. 300*

BARNESS, L. A. 295*, 388

Barrett, C. T. 289*

Battaglia, F. C. 193

Baughan, M.A. 311 *

BEARD, R.W. 431*

Beaudry, P. H. 400*

BECKER, F. B. 291*

Behavior 421*

- development $290 *, 371$

- lateralizing 243

BELD, J.W. 316*

BELL, J.J. 43

Bellanti, J. A. 298*

Belsey, M.A. 315*

BERAN, A. V. 424*

BERGER, C.K. $301 *$

Bergstrom, W.H. 408*

BERKOVICH, S. 314*

Berman, J. L. 404*

Bernstein, J. 319*

BERRYhILL, W.S., Jr. 264

BeZan, A. I. 312*

Bicarbonate 193

- transport 408*

Bile acids 293*

Bilirubin 237, 307*, 361

Bingol, N. 320*

BIRCH, H. G. 243

Birth trauma 243

Bladder 396*

BLAESE, R.M. 300*
BLANKenship, W.J. 317*

Blastogenesis 418*

Blast transformation 209

Blizzard, R. M. 501

BLOCK, J. 317*

Blood 416*

- cord 187, 493

- pressure $316^{*}$

BLoom, G.E. 297*

Bone marrow 299*

Bongiovanni, A. M. 291*, 309*

Bourtasin, J.D. 313*

Bowe, E. T. 399*

BradLey, K. H. 441

Brain 305*, 307*, 352, 420*, 431*

BRANSOME, E.D., Jr. 306*

Bray, P.F. 250, 254

BRENT, R. L. 291*

BRIDGES, R. 298*

Bruton's disease 209, 292*

BRYSON, M.F. 308*

BuCKLEY, R.H. 72, 299*

BuEscher, E. L. 298*

BYRNE, P.A. $316^{*}$

Cadman, T.E. 420*

Calcagno, P.L. 332

Calcium, muscle 303*

Calciferol 408*

Caloric restriction 307*

Camacho, A. M. 309*

Cancer 406*

Candida albicans 7

- antigen 209

Carbohydrate absorption $301 *$

Carbon monoxide $313 *$

Carboxyhemoglobin 313*

Carey, J. 293*

Carotid sinus 264

Carver, M.J. 305*

CASH, R. 301*

Castells, S. 306*

Cat's Eye syndrome 297*

CAtz, C.S. $291 *, 361$

Cave, P. 289*

Cell biology 356

Central nervous system 243, 250

Cerebral circulation 420*

Chapman, S.S. 409*

Chase, H.P. $420^{*}$

Ghayoth, R. 302*

Cheek, D. B. $310^{*}$

Chen, Y.C. 479

Crrilds, B. 414*

Cholesterol 172

Chondrogenesis 85

Chondroitin sulfate 405*

Cimristran, C. L. 300*

Chromosomes 296*, 297*, 486

Circulation, pulmonary 278*

Clark, L. C., Jr. 424* 
Gleveland, W.W. 308*

Coagulation 319*

Cochran, W.D. 317*

Cohen, M. M. 296*, 486

Colchicine 294*

Colle, E. 311*

Colombo, J.P. 464

Colostrum 103

Complement 300*

Computer 290*

Conly, P.W. 308*

Conte, F.A. 43

Convulsions 393*

Copenhaver, J. H. 305*

Cornblath, M. 294*, 301*

Coronary artery disease $296^{*}$

Corpus, M.A. 287*

Corticords, urinary 501

Corticosteroids $312 *$

Corticosterone 454

Cortisol 85, 456, 501

Cortisone 451

Coscia, A. 451

Costin, G. 310*

Craig, J. 316*

CRAWFord, J.D. 302*

Creatinine 17

CRIGler, J.F., Jr. 310*

Gunningham, G. C. 404*

Cyanosis 289*

Gystathionase 149

Cystathionine 149

Cystathionine synthatase $403 *$

Cysteine 441

Cystic fibrosis of the pancreas $292 *, 293 *, 295 *, 378,464$

Cystine 149, 441

Cystinosis 441

Cytomegalovirus 314*, 418*

Cytosine arabinoside $312 *$

DAILY, W.J. 289*

D'Albora, J. B. 332

D'Amodio, M.D. 415*

DANIEL, S. S. 395*

Davidson, W. 426*

Davis, C. $297 *$

Davis, H. T. 317*

DAx, R. W. 404*

DEASY, P.F. 332

DeGastro, F.J. 292*

Deferoxamine $312 *$

DeLamerens, S.A. 312*

DeLemos, R. 317*

Demyelination 304*

Der Kaloustian, V.M. 414*

Dermatitis $301 *, 315^{*}$

DeSousa, R. C. 22

Developmental biochemistry $187,291 *, 293 *, 294 *, 302 *$ $305 *, 307 *, 318 *, 352,361$, $412 *, 413 *, 415 *$

Developmental pharmacology 29, 514

Developmental physiology 421 *

Diabetes

- insipidus $397 *$

- mellitus 94, 198, 300*, 301*, $422 *, 426 *$
Diarrhea 293*, 316*

Diazoxide $310 *$

Dickson, M. 198

Diczfalusy, E. 413*

Diet $426 *$

Dietary protein 131

Differentiation 294*

- cellular 412*

DiGeorge, A. M. 295*

Digitalis 430*

Dihydroorotase 307 *

Diphtheria 315*

Disaccharidase 292*

DiSant'Agnese, P.A. 295*

DNA $352,410 * 451$

- polymerase $306^{*}$

Dobrins, W. T. 303*

Dolman, G. L. 304*

DORFMAN, A. 405*

Down's syndrome $304 *$

Drachman, H. 315*

Drash, A. 94, 397*

Dray, R. 456

Drug distribution 29

Drummond, K. 292*

Dubois, R.S. 292*

Duc, G. 119

Duncan, B. $301 *$

DunN, H. G. 304*

Dwarfism

- constitutional 43

- psychosocial 43

Dysgammaglobulinemia 7

Dyspnea 289*

EberLein, W.R. 309*

Echo virus 314*

E. Coli 316*

Edelmann, G. M., Jr. 421 *

EDSON, J.R. 319*

EDWARDs, J.E. 288*

Electroencephalogram 305*

Elema, J. D. 519

ELLIS, E. F. 300*

Embryo 307*, 308*

EMrICH, H. M. 464

Encephalitis 313*

Encephalopathy 303*

Endoplasmic reticulum 294*

Energy production 161

ENGEL, R. R. 313*

Enteropathogenic agents $316 *$

Enuresis 396*

Enzymes

- conformation $307 *$

- hepatic 294*

- intestinal 294*

Epidemiology 316*

Epinephrine 318*

ERKAN, V. 317*

ERLANDSON, M.E. 312*

Erythrocyte 172, 302*, 311*,313*

- lipid 172

ESPERANCA, M. 396*

ESTERLY, J.R. 294*

Estradiol 412*

Estrogen 305*

Evoked potentials $305^{*}$

Exchange transfusion $312 *$
FARQUHAR, J. W. 198

Fatty acids $172,301 *, 305 *$

FeKete, M. 161

FERGuson, C. 288*

Ferrous sulfate 479

$\alpha$-Fetoprotein $318 *$

Fetus 289*, 293*, 296*, 318*, $399 *, 486,493$

Fibrinogen $311 *, 319 *$

Field, J.B. 94

Finkelstein, J.W. 501

FINSTER, M. 399*

FISH, I. 307*

Flanigan, W.J. 321*

FLETCHeR, G. 289*

Folate reductase $306 *$

Folic acid 179, 312*

Follicle stimulating hormone 308*

Forman, E. N. $311 *$

Frankenburg, W.K. $301 *$

FRASIER, S. D. 305* 310*

FreIER, E. 298*

Friedman, C.J. 371

Friolet, B. 464

Frosah, W.A. 296*, 486

FRY, L.R. 295*

Fulginiti, V.A. 292*, 299*, 314*

Galactosidase 294*

GALOFRE, A. 307*

GANDY, G.M. 416*

Gangliosides 304*

GARGES, L.Y. 397*

Gastrostomy 294*

Gaull, G.E. 403*

Genetics 296*, 306*, 311*, 414*, $417 *, 486$

Gerald, P.S. 297*

Germ-free state 301*

Gerrard, J.W. 396*

GERRITSEN, T. 254

Gessner, I.H. 308*

Gewurz, H. 300*

Giambetti, P. 303*

Gibson, G.W. 479

Gibson, R.M. 292*

Gilbert, E. F. 299*

GilladoGA, A.C. $287 *$

GrTlin, D. 399*

Glasgow, A. M. 319*

Glasgow, L. A. 298*

Gliadin 298*

Globulin

$-\alpha-1298 *$

$-\beta_{1 \mathrm{C}} 320^{*}$

- $\gamma 320^{*}$

- macro 314*

Glomerulus 271

Glucagon 94, 318*, 426*

Glucogenesis $302 *$

Glucose 198, 287*, 295*, 309*, $318 * 319 *$

Glucose Kt 198

Glucuronidase 294*

Glucuronide 36

Glutathione 441

Glycerol 309*

Glycine 254, 295*, 320* 
Glycogen $415 *$

- storage disease $302 *$

Glycolipids 304*

Glycoprotein 298*

Glycosuria 319*

GODARD, G. 22

Goldman, A.S. 103, 306*

Goldstein, A. 297*

Goldstein, G. B. 298*

Gonad 308*, 309*

Gonadal dysgenesis 43

Gong, L. 254

Good, R. A. 300*, 319*

GOODFRIEND, L. 298*

Gorden, P. 295*

Gordis, L. 290*

GotofF, S.P. 209, 311 *

Granulomatous disease $315 *$

Graven, S. N. 318*

Graves, M. 304*

Graystone, J.E. 310*

Graziani, L.J. 305*

GREenberg, R. E. 302*, 412*

GREENE, R.A. 289*

GREGG, J.R. $416^{*}$

GriffIN, C. A., II 1264

Groeschel, M.M. 486

Grossman, H. 287*

Growth 17, 451

- acceleration 309*, 397*

- cellular $310^{*}$

- failure $305^{*}$

- hormone 43, 94, 295*, 305*, $310 * 311 *$

- retardation 43, 307*, 308*, $310^{*}, 311^{*}$

- retardation, intrauterine $17,415^{*}$

Grumbach, M. M. 43

Gunn strain rats 237

Guntheroth, W. G. 288*

GYÖRGY, P. 389

HAgGerty, R. 392*

Hait, G. 287*

HALler, A. $317 *$

Halloran, K. H. 430*

Hallucinogens 486

HANDWERGER, S. 295*

Hanley, W.B. $426^{*}$

Hanshaw, J.B. 314*

Haque, N. 300*

HARNED, H.S., Jr. 264

HARPER, P.A. 110

HARRIS, R. C. 403*

HART, R. 301*

HASSELMEYeR, E. G. 316*

Hattler, B.G., Jr. 299*

HaworTH, J. G. 17

HAWORTH, S. G. 395*

Heagarty, M. 392*

Health care $316^{*}$

Health services 290*, 291*, 392*, $402 *$

Heart

- disease, congenital 287*, 288*, $289 *, 297 *, 308 *$

- failure $287 *$

- murmur 394*

- output 289*
Heart rhythm 288*

Heiner, D. C. 254, 298*

HELFER, R.E. 290*

Hemodynamics 479

Hemoglobin 193, 311*, 417*

- fetal $312 *$

- sickle $313 *$

- synthesis 296*, 312*

Hemophilia 313*

Hemophilus influenza $410 *$

HENNEY, C.S. 300*

Heparin 319*, 378

Herbst, J.J. 294*

Herdman, R. C. 319 *

Hereditary disease 72, 519, 525

HeRRINGTON, R.T. 264

Hexobarbital 291*

Heymann, M. A. 289*

Hillman, D. A. 311 *

Hillman, R. E. $320 *$

HirschHORN, K. 296*, 297*, 486

HobBins, T.E. $314 *$

Hohenauer, L. 415*

Hollerman, G.E. 332

HoLt, L. E., Jr. 131

Home care 291*

HommEs, F.A. 519

Homocystinuria 149

Homoserine 149

Honig, G.R. 311 *

Hopps, H.E. 314*

Hormones, pituitary 310 *

Houck, P.W. 319*

Hsia, D. Y. Y 404*

Hsu, L, Y. 297*

Hurler's syndrome 405*

HuRwitz, R. 294*

HUTTENLOCHER, P.R. 303*

Huxtable, R.F. 424*

Huygelen, C. 38

Hyaluronic acid $405 *$

Hydrochlorothiazide 22

Hydrocortisone 420*

$\beta$-Hydroxybutyrate $309 *$

Hydroxyindole-o-Methyltransferase 293*

Hydroxymethyltetrahydrofolate 254

Hydroxyproline 17

$3 \beta$-Hydroxysteroid dehydrogenase 306*

Hyperaminoacidemia $302 *$

Hypercapnia 430*

Hypercholesterolemia 296*

Hyperglycinemia, nonketotic 250,254

Hyperoxia 420*, 429*

Hypersensitivity, delayed 209, $299 *, 300 *, 314 *$

Hypogammaglobulinemia 72, $292 *, 299 *, 300 *$

Hypoglycemia 43, 287*, 305*, $310^{*}$

Hypopituitarism 308*, 397*

Hypoxanthine guanine phosphoribosyl transferase $414 *$

Hypoxia 288*, 430*
Immunity $38,103,314 *, 407 *$, $409 *$

- cellular 299*

Immunodeficiency 299*

Immunoglobulins 7, 72, 298*, $300 *, 407 *$

Infants 38,131

Infection 409*

- chronic $315^{*}$

- recurrent 7, 419*

- viral 298*,313*

Inherited disease $406 *$

Insulin 43, 94, 198, 287*, 295, $301 *, 309^{*}, 310^{*}, 399 *, 422 *$, $426 *$

Intelligence $371,426 *$

- quotient 110, 292*

Interferon 298*

Intestine $293^{*}, 294^{*}$

IRIAS, J. 302*

Iron $311 *$

- deficiency 179

- poisoning $312 *, 479$

ISLES, T.E. 198

Isoproterenol 289*

Isozymes 296*, 311*

ITANO, H.A. 296*

JamES, L.S. 395*, 399*

JANEWAY, C.A. 7

JANSEN, J.F. 519

JANSONS, R.A. 310*

JARAI, I. 161

Jaundice 237

Jayalakshmi, P. 304*

Jehle, J.W. 420*

JonXIs, J.H.P. 519

KAN, Y.W. 417*

KAPLAN, E.L. 409*

Kaplan, S.A. 310*

Kaplan, S.L. 43

Karzon, D.T. 407*

KASPAR, J. C. 291 *

KATURICH, N. 424*

Katz, M. 313*

KAYE, R. 300*, 419*

KAZAZIAN, H.H., Jr. 296*

KeKomaki, M. 307*

KeMPe, C.H. 314*

KenNedy, C. 420*

KENNY, F.M. 94, 397*, 501

KERR, G.R. 187, 493

Ketoacidosis $300^{*}$

16 -Keto-androstenediol $413 *$

Kidney 22, 271, 302*, 332, $421 *, 525$

- biopsy $318 *$

- blood flow 332

- disease $319^{*}, 320 *, 321 *, 408^{*}$

- disease, experimental 319*, $320 *$

- function $320 *, 332$

- tubules $320 *, 408 *$

KING, K. C. $422 *$

Krtahen, H. 215

Kratskin, G. 303*

KNIKER, W.T. 321*

Kohrman, A. 412 * 
KOLER, R.D. 296*

KOMrOWER, G. M. 149

KoprowsKI, H. 313*

Korein, J. 290*

Korns, M. E. 288*

KosA, J. 392*

KoWARSKI, A. 501

Krabbe's sclerosis $304 *$

KRASNER, J. 307*

Kretchmer, N. 283, 294*, 307*

KRETSCHMER, R. 7

KRILL, C.E. 313*

KRIvIT, W. 293*, 302*

Kruger-Thiemer kinetics 29

KuIPERS, J.R. G. 519

KURY, G. 316*

Lactase 292*

LAKE, B. D. 304*

LAMARRE, F.R. 287*

LAMMOT, T.R., III $315 *$

LAMPKIN, B. C. 312 *

LANCASTER, J.R., Jr. 299*

LANE, D.M. 312*

LANG, D.J. 418*

LANZKOWSKY, P. 312*

LARDINOIS, R. 318*

LARSON, H. E. 314*

LAWRENCE, A. M. 426*

LAzARovitz, E. 302*

LEASH, A. 292*

LEBLANG, D. 315*

LEDUC, J. $317 *$

LEE, L. A. 308*

LEGRAND, J. C. 456

LeIBy, G. $317 *, 410$ *

LEIKIN, S. 179, 312*

Lesch-Nyhan's syndrome $414 *$

Leukemia $312 *, 313 *, 356$

Leukocytes $304 *, 315 *$, 418*, $419 *, 441,486$

Leukodystrophy, globoid cell 304*

LEVIN, A.R. 287*

LEVIN, S. 302*

LEVINE, H.D. 294*

LeVitsky, L. $419 *$

LEVY, H. L. 302*

LEWY, J.E. 318*

Lr, M.M. 311*

LuebMan, J. 292*, 394*

Life expectancy $291 *, 292 *$

LINDE, L. M. 288*

LINDLEY, A. 311*

LING, S. M. 310*

LiNsAo, L. S. 426*

Lipoproteins 296*

LISCHNER, H.W. $315 *$ * 418*

Lithocholic acid 293*

LitTLEFIELD, J. W. 306*

Liver 294*, 298*, 307*, 361, 514

- cirrhosis 293*, 298*

- disease $303^{*}$

- fatty 519

LORIDAN, L. 309*

LOVRIEN, E.W. 296*

LOWDEN, J.A. 125

Lowman, J. T. 302*

LuBs, H.A. 297*
LuCAS, Z. J. 299*

LUKEMEYER, J. W. 301*, 318*

Lung 119, 287*, 288*, 316*, $317 *, 429 *$

- elasticity 119

Luteinizing hormone 309*

LYMAN, M. 290*

Lymph node 7

Lymphocytes 7, 103, 209, 299*, $300^{*}, 313^{*}, 418^{*}$

Lymphocytotoxicity 7

Lymphoid tissue 294*

Lysergic acid 296*, 486

Lysine 525

Macaca mulatta 187, 493

MacCReady, R.A. 302*

MacGillivray, M. 407*

MacKinney, L.G. 264

Macrophage 103, 298*

Madigan, P. M. 302*

Madsen, J.A. 250

MAKowski, E.L. 193

Malabsorption, intestinal 293*, $312 *$

Malate dehydrogenase 296*

Malignancy 291*, 406*

MaLinow, M.R. 296*

Malnutrition 292*, 311*, 426*

Mancuso, S. 413*

MANGOS, J.A. 293*, 378

Marfan's syndrome $405^{*}$

MARKowITZ, M. 290*

MARs, G. 29

Martin Du PAN, R. 38

Marubini, E. 29

Masland, W.S. 313*

Matalon, R. 405*

Maternal deprivation syndrome 43

MaUer, A. M. 312*

MaAdams, A. J. 320*

MaAllister, R. M. 356

MaCollum, A.T. $402 *$

MaCoy, E. E. 304*, 319*

MaInTIRe, M.S. 421*

MCINTYRE, O.R. $311^{*}$

MaKhann, G. M. 304*

MaKINLEY, F.W. 410*

MCShERRY, N.R. 293*, 378

Measles 314*

Meconium 293*

Megaloblastosis 179, 312*

Megevand, A. 22

MeILE, R.L. 421*

Melatonin 293*

Mellman, W.J. 303*

Membrane transport 464, 525

Menarche 308*

Menkes, J.H. 305*

Mental age 292*

Mental retardation 110,250 , $297 *, 301 *, 305 *, 371,404 *$, 426*

Mergenhagen, S.E. 300*

Merrill, D. 292*

Meschia, G. 193

Mesel, E. 289*

Mesenchymal cells 85
Mestyan, J. 161

MetaLolic disease 519, 525

Metabolism

- amino acid $320^{*}$

- calcium 303*

- carbohydrate $319^{*}, 415^{*}$

- drug 291*

- electrolyte $287 *$

- glucose 295*,301*,310*,399* $422 *, 426 *$

- glycogen $302 *$

- protein 305*, 306*, 320*

- urea $307^{*}$

Metcoff, J. 318*, 320*, 415*

Methimazole $420^{*}$

Methionine 302*

Methioninemia 403*

Methylmalonic acid 295*

Meyer, H. M., Jr. 314*

Microorganisms 410*

Microsomes 294*, 514

Micturition 396*

Migeon, B.R. 414*

Migeon, C.J. 501

Milk, human $301^{*}$

Miller, D. R. 311*

Miller, M.E. 419*

Miller, R. W. 406*

Mintz, D. 94

Mitochondria 271

Moes, C. A. F. 426*

Moffett, B. C. 295*

MolLER, J.H. 288*

MoloshoK, R.E. 297*

Momma, K. 288*

Moniliasis 299*

Moore, R.Y. 293*

Moreau, T. 243

Morgan, B. G. 288*

Morgan, W. $317 *$

Morrison, T. 308*

Morrow, G. III 295*

Mosaicism 297*

Moses, S. W. 302*

Moshang, T., Jr. 309*

Mosier, H.D., Jr. 310*

Movassaghi, N. 312*

Mucopolysaccharides 405*

Mucosa, intestinal 292*, 294*

Muller, A.F. 22

Mutation, bacterial 306*

Myeloid bodies 271

MYers, R.E. 431*

Myogenesis 85

NAGHMAN, R. 317*

NAFF, G. $300^{*}$

NAGAO, T. 312*

NANCE, J. L. 304*

Nathan, D. G. 417*

NeERHout, R.C. 172

Neoplasia 356

Nephritis 320 *

- glomerular 318*,320*

Nephrogenic diabetes insipidus 22, 271

Nephrosis $321 *$

Nerve growth factor $304 *$

Neurolipids $420 *$ 
Neuropathy 304*

Newborn 17, 125, 198, 243, 264, $293 *, 295 *, 297 *, 301 *, 305 *$ $307 *, 308 *, 312 *, 315 *, 316 *$ $317 *, 318 *, 395 *, 399 *, 400 *$, $420 *, 422 *, 424 *, 431 *$

Newell, K.W. 316*

Nicolas, J.G. $430^{*}$

NIERnBerG, M. M. 193

Noren, B. 418*

Norepinephrine 293*, 317*

NorTon, P.M. 131

Nutrition 131, 187

NyHAN, W. L. 250, 254, 414*

Nystagmus 304*

Obesity 426*

Odeli, G.B. 237

OGRA, P.L. 407*

OH, W. 415*

Oliver, T.K., Jr. 315*

Oppex, W. C. 110

Opsonin 419*

Optic atrophy $303 *$

Ornithine 525

Ornithine-ketoacid aminotransferase $307^{*}$

Ortho-aminophenol 361

OUTERBRIDGE, E. W. 400*

Oxidative phosphorylation 320 *

Oxygenation 289*, 424*

Oxygen dissociation curve 193

Oxygen toxicity $316 *, 317 *$

Oxytocin $431 *$

Page, L.A. 318*

Paglia, D.E. 311 *

Panhypopituitarism 43

Papadopoulou, Z. L. 332

Parkman, P.D. 314*

Parotid gland 293*, 388

Parturition 399*

Paulsen, E. P. 426*

Pearson, B. 294*

Peetermans, J. 38

Peller, J. 293*

Peritoneum 416 *

Perletti, L. 29

Peyer's patches 299*

pH 193

Phagocytosis 315*, 418*, 419*

Phansalkar, S.V. 131

Pharmacokinetics 29

Pharmacology, developmental 29

Pharmacometabolism 514

Phenylalanine 404*, 426*

Phenylketonuria $301 *, 371$, 404*, 426*

- maternal 301*

Phospholipids 172, 302*, 304*, 318*

Phosphorus 125

Pickering, R.J. 300*

Pildes, R.S. 294*, 301*

Pineal gland 293*

Pirani, C. $318 *$

Pituitary gland $310^{*}, 514$

Placenta 293*, 399*, 413*

Plasma 419*
Plasma aminogram 131

- storage $311 *$

Platelets 179

Pneumonia 288*

Pogue, R. G. 292*

Poisoning 421*

Poliovirus 407*

Polypeptides 378

Poppers, P. 399*

Potassium

- citrate $303 *$

- exchangeable 22

Prealbumin 318*

Precocious puberty $309 *$

Pregnancy 493

Premature infant 161

Prematurity 17, 110, 294*, 305*, $317 *, 400^{*}$

PrICHARd, J.S. 125

Prinzie, A. 38

Progesterone 308*

Prolactin 397*

Propionic acid 519

Propionyl coenzyme A carboxylase 519

Propranolol 289*

Protein

- dietary $302 *, 421 *$

- specific dynamic action 161

- synthesis $412 *$

Psychological testing 110

Psychometry 292*

Pulmonary compliance 119

Pulmonary resistance 119

Pulmonary stenosis 288*

Purugganan, G. G. 179, 312*

Pyelonephritis 332

Pyoderma 409*

Pyridoxine 149

Pyrimidine biosynthesis 307 *

Pyruvate kinase $311^{*}$

RAFFENSPERGER, J.F. 294*

RÄIHÄ, N. C. R. 307*, 399*

Ramos, A.D. $317 *, 400 *$

RAO, N.V. 288*

RAPPAPORT, R. 456

RAstogi, R.N. 125

Reagin 298*

Receptor, beta 289*

REED, C.F. 311 *

REED, G. 356

Renal blood flow 332

Renal function 332

Renin 22

Respiration 264, 298*

Respirator, negative pressure 400*

Respiratory center 264

Respiratory distress syndrome 289*, 308*, 317*, 318*, 400*, 424*, 429*

Reticulocytes $313 *$

Rev-KuRY, L.H. 316*

Reye's syndrome 303*

Reynolds, J.W. 413*

Rheumatoid factor $300^{*}$

RICHTERICH, R. 464

Rickets 408*
RIDER, R. V. 110

RighthaND, F. 407*

RNA 352, 451

Robertson, L. 392*

RODER, R. 315*

RODKEX, F.L. 313*

Rodriguez, H. 293*

Roe, G.R. 306*

RoITMAn, E. 131

Root, A.W. 309*

Rorke, L.B. 313*

Rose, B. 298*

Rosen, F.S. 7

ROSENBERG, L.E. 320*

Ross, A. 385

Rossi, E. 464

Roth, J. 295*

Roy, C.C. 292*

ROYER, P. 456

Rubella 38, 314*

Rubinstein, D. 302*

RuddLe, F.H. 297*

Rudolph, A. M. 289*

Salicylism 303*

SAlinas-Madrigal, L. 318*

Saliva 293*, 378

Salivary gland 293*

Salt-losing syndrome 456

Salt metabolism 464

Salvador, H.S. 416*

SANDBERG, D.H. 308*

SARGia, S.R. 395*

SAY, B. M. 297*

SCHAFFER, D. 304*

SCHAFFER, J. J. 301*, 318*

Schain, R.J. 305*

Schalch, D.S. 308*

SchimpfF, S. 430*

Schneeman, F. 311*

SCHNEIDER, J.A. 308*, 441

Schubert, E.T. 287*

SCHUBERT; W.K. 311 *

Schulman, I. $311^{*}$

Schulman, J. L. 291*

Schwartz, A.D. 303*

Schwartz, A.H. 402*

Schwartz, C. H. 288*

Schwartz, E. 417*

SchWARTz, R. 399*, 422*

SchWARz, V. 149

ScotT, T.F.M. 304*

SCRIVER, C.R. 525

SEEGMILleR, J. E. 308*, 441

SEGAL, S. 316*

SEGER, S. $311^{*}$

Seizures 250, 421 *

SENIOR, B. 309*

Sereni, F. 29

Serine 149, 254

Sharp, H. 293*, 298*

SHEPARD, T.H. 295*

ShePARD, J.T. 287*

Shock 479

Sibinga, M.S. 371

Sickle cell disease $313 *, 315 *$

SidBuRY, J. B., Jr. 72

Sideroblast $311^{*}$

SinclaIR, M. 315* 
Sinnamon, H. M. 371

Sissman, N.J. 289*

Slater, C. H. 290*

Sleep 305*

SLONE, D. 310*

SmITH, C.A. $317 *$

Smith, C. W. 103

SMith, F. G., Jr. 305*

SMITH, M.H.D. 316*

Smith, R.A. 293*

SMITHWICK, E.M. 314*

Snipes, C. A. 501

SNyderman, R. 300*

SNyderman, S.E. 131

Sodium metabolism 378

Sodium, transport 293*

Sokoloff, L. $420 *$

Solomon, G. 403*

Solvonuk, P.F. 416*

Sood, S. 394*

SoYKA, L. F. 294*

Spasmus nutans $304 *$

Spastic quadriplegia $303 *$

Spaulding, J.S. 501

Specific dynamic action of protein 161

Spitzer, R. E. 320*

STACHURA, I. 410*

Srahlman, M.T. 317*

Standen, A. C. 294*

Staphylococcus 315*

STEINER, M. 314*

STEINFELD, H.J. 314*

STEISEL, I. M. 371

Stern, L. 317*, 400*

Steroidogenesis $413 *, 501$

STIEHM, E.R. 319*

Stoll, E. 464

Strauss, J. 424*

Streptococcus $409 *$

Strober, W. 300*

2-Sulfa-3-methoxy-pyrazine 29

Sulfatide $420 *$

Sulfonamide 29

Sulfonamidophenethanolamine $300 *$

Sulianti, J. 316*

SUNSHINE, P. 289*, 294*

Súsen, A. $397 *$

Sweat

- gland 464

- rate 464

Talner, N.S. 430*
Tenenhouse, A. 303*

Teramo, K. 399*

Teratogenesis 85

Teratology. 306*

Testosterone 309*

$\alpha$-Thalassemia $417 *$

THAM 193

Thermal energy needs 161

Thermogenesis $317 *$

Thymus 294*, 299*, 451

- dysplasia 292*, 299*, 418*

Thyroid hormones $420^{*}$

TICK, L.J. 290*

Tina, L.U. 332

Tissue culture 356

Tolbutamide 94, 295*

TORRES-PINEDO, R. 293*

TOWELL, M. E. 416*

Transfusion, intrauterine 416 *

Trouy, R. L. 309*

Trygstad, C. W. 319*

Tuberculosis $314 *$

TuakeR, S.H. 304*, 313*

Tumor 514

Turkewitz, G. 243

Turner's syndrome 297*

Tutrle, A.H. $312 *$

Tyrosinosis $403^{*}$

UDP-glucuronyl transferase 307 *

UMANSKY, R. 85

Umbilical vein $317 *$

Urea transport 237

Uremia 319*

Uric acid $414 *$

Uridine phosphoglucuronyltransferase 361

Urine osmolality 237

USHER, R. 429*

Vaccine 38

- measles 314*

- polio 407*

- rubella $314 *$

Vagina $412 *$

Valentine, W.N. 311 *

VAN DEN BERG, B.J. 393*

Vasopressin 22

Vaughn, K. L. 292*

VAZQUEZ, A.M. 94

Vena cava 288*

Vengusamy, S. 294*

Ventilation, artificial 289*

Verbo, S. 486
VICK, N.A. $293 *$

VILES, P.H. 287*

Vincristine $312 *$

Viremia 418*

Virus 38

Vital capacity 119

Vitamin $B_{12}$ 179, 295*

Von Bogaert degeneration $303 *$

Vossough, P. 179

WaLdMANn, T.A. 300*

Walravens, P.A. 420*

WANNAMAKER, L. W. 409*

WARRNER, R. 301*

WARWICK, W.J. 292*

WASSERMAN, E. 320*

Weaver, J.A. 293*

WEIDMAN, W.H. 287*

WeitzMAN, E.D. 305*

WEST, C. D. 320*

WHELAN, D. T. 525

Whirte, C.J. 314*

White, J.G. 313*

Whitten, G.F. 479

WIENER, G. 110

WILKINS, J.L. 297*

WILKINSON, H.A. 317*

Williams, C.P.S. 315 *

WILSON, J. 304*

Wilson, J.T. 514

WINICK, M. 307*, 352, 451

WinkeLSTEIN, J.A. 315*

Wiskott-Aldrich syndrome $300^{*}$

Wrovist, N. 413*

WrTtig, H.J. 299*

Wolfish, N.M. 421 *

WOLFSDORF, J. 317 *

WOLINSKY, J. 311 *

WONG, P.K.W. 149

X-irradiation 291*, 310*

YAFFE, S. J. 291*, 307*, 361

YAP, L.L. 415*

YeRUSHALMY, J. 393*

Yoshida, T. 320*

Young, W.J. 414*

Younoszai, M.K. 17

ZEE, D.S. 296*

ZINKHAM, W.H. 296*

Ziter, F.A. 250

ZMIJEWSKI, G. M. 299* 